\title{
Resonant inelastic x-ray scattering at the limit of subfemtosecond natural lifetime
}

\author{
T. Marchenko, ${ }^{1,2, a)}$ L. Journel, ${ }^{1,2}$ T. Marin, ${ }^{1,2}$ R. Guillemin, ${ }^{1,2}$ S. Carniato, ${ }^{1,2}$ M. Žitnik, ${ }^{3}$ \\ M. Kavčič, ${ }^{3}$ K. Bučar, ${ }^{3}$ A. Mihelič, ${ }^{3}$ J. Hoszowska, ${ }^{4}$ W. Cao, ${ }^{4}$ and M. Simon ${ }^{1,2}$ \\ ${ }^{1}$ Laboratoire de Chimie Physique - Matière et Rayonnement, Université Pierre et Marie Curie, \\ UMR 7614, 11 rue Pierre et Marie Curie, F-75231 Paris Cedex 05, France \\ ${ }^{2}$ CNRS, Laboratoire de Chimie Physique - Matière et Rayonnement, UMR 7614, 11 rue Pierre et Marie Curie, \\ F-75231 Paris Cedex 05, France \\ ${ }^{3}$ J. Stefan Institute, Post Office Box 3000, Ljubljana SI-1001, Slovenia \\ ${ }^{4}$ Department of Physics, University of Fribourg, CH-1700 Fribourg, Switzerland
}

\begin{abstract}
We present measurements of the resonant inelastic x-ray scattering (RIXS) spectra of the $\mathrm{CH}_{3} \mathrm{I}$ molecule in the hard- $x$-ray region near the iodine $\mathrm{L}_{2}$ and $\mathrm{L}_{3}$ absorption edges. We show that dispersive RIXS spectral features that were recognized as a fingerprint of dissociative molecular states can be interpreted in terms of ultrashort natural lifetime of $\sim 200$ attoseconds in the case of the iodine L-shell core-hole. Our results demonstrate the capacity of the RIXS technique to reveal subtle dynamical effects in molecules with sensitivity to nuclear rearrangement on a subfemtosecond time scale.
\end{abstract}

\section{INTRODUCTION}

The evolution of x-ray scattering techniques, successively, progressed along with the development of new x-ray radiation sources. The availability of hard-x-ray radiation advanced, among the rest, the field of nonresonant fluorescence spectroscopy. The underlying mechanism here involves innershell ionization and a radiative decay, which can be well described as a two-step process, so that the resulting fluorescence spectrum is independent of the incident X-ray radiation properties. In contrast, an inner-shell excitation below the ionization threshold results in X-ray emission spectra sensitive to the energy and bandwidth of the incident radiation. In this case, the absorption and emission processes cannot be treated separately and are described by a single transition amplitude. The physical process behind the resonant inelastic X-ray scattering (RIXS) consists of a resonant excitation, in which an inner-shell electron is promoted to an unoccupied orbital, leaving a hole in the core shell, coupled to a relaxation of the core-hole via $\mathrm{x}$-ray photon emission.

Resonant $\mathrm{x}$-ray emission spectroscopy proved to be a compelling technique for studying electronic properties of various systems. Being well established as a key tool for revealing the electronic properties of solids, ${ }^{1}$ RIXS has also remarkably advanced in spectroscopy of isolated atoms and molecules using third generation synchrotron radiation facilities. For example, it was demonstrated that a dynamical symmetry breaking in molecules occurring on the time scale of the core-hole lifetime can be studied due to the symmetry dependence of the $\mathrm{x}$-ray emission intensities on the selection rules. ${ }^{2,3}$ Polarization and anisotropy of $\mathrm{x}$-ray emission can be used to study orbital components, bond directions, and molecular orbital symmetries. ${ }^{4-6}$ Recently, molecular-field effects

\footnotetext{
a) Author to whom correspondence should be addressed. Electronic mail: tatiana.marchenko@upmc.fr.
}

on the electronic structure of isolated molecules have been observed in the polarization dependence, ${ }^{7,8}$ double ionization cross section, ${ }^{9,10}$ and electronic state interferences. ${ }^{11}$

Besides the remarkable sensitivity of RIXS as a probe of the chemical environment of the selected atom in a molecule, a complementary insight into the interaction of molecules with external radiation can be obtained considering the dynamical aspect of the RIXS spectroscopy. Our previous studies on chlorine compounds showed that RIXS spectral features carry information on nuclear dynamics in a molecule upon core-hole excitation. Nonlinear dispersion and a significant narrowing of inelastic emission lines around the resonant excitation were shown to reflect molecular bond elongation. ${ }^{12,13}$ The time scale of the nuclear dynamics, occurring in competition with the core-hole relaxation, is determined by an effective photon scattering time reaching its maximum value, the natural lifetime of the core-hole exactly at the resonance energy. ${ }^{14,15} \mathrm{An}$ analysis based on $a b$ initio potential-energy calculations and simulations of the wave packet propagation along the potential-energy surfaces upon resonant excitation of the $\mathrm{Cl} \mathrm{K}$-shell demonstrated the sensitivity of RIXS spectral features to the nuclear motion on a sub-Ångström scale in the time interval set by the natural lifetime of $1 \mathrm{fs}$. Pushing further the time limit we aim to challenge the capacity of the RIXS technique as a tool to detect subtle dynamical effects in molecules.

\section{EXPERIMENTAL DETAILS}

In this paper we present results of RIXS measurements of the gas-phase iodomethane $\left(\mathrm{CH}_{3} \mathrm{I}\right)$ performed at the ID26 beamline at the European Synchrotron Radiation Facility (ESRF). The experimental setup is described in detail in Ref. 16. Essentially, a linearly polarized focused beam with $250 \times 50 \mu \mathrm{m}^{2}$ cross section passes through the stainless 
steel gas cell separated from the vacuum by $12.5 \mu \mathrm{m}$ thick kapton windows and filled with $\mathrm{CH}_{3} \mathrm{I}$ vapor at pressure of $\sim 400$ mbar. The sample absorption was measured by a photodiode after the beam has passed through the gas cell. Scattered photons were collected in the polarization plane at $90^{\circ}$ with respect to the direction of incoming light employing a crystal spectrometer in Johansson geometry with a $500 \mathrm{~mm}$ Rowland circle radius. The second order reflection of a quartz (1010) crystal was used and the diffracted photons were detected with a thermoelectrically cooled $\left(-40^{\circ} \mathrm{C}\right) \mathrm{CCD}$ camera with $22.5 \times 22.5 \mu \mathrm{m}^{2}$ pixel size. The incident radiation with full width at half maximum (FWHM) of $\sim 0.14 \mathrm{eV}$ was tuned with a step of $0.2 \mathrm{eV}$ close to $\mathrm{I}_{2}(4849-4864 \mathrm{eV})$ and $\mathrm{L}_{3}(4555-4569 \mathrm{eV})$ edges across the energy region of $2 p_{1 / 2,3 / 2}$ resonant inner-shell excitation. Three series of RIXS spectra were acquired corresponding to the $3 d_{3 / 2} \rightarrow 2 p_{1 / 2}\left(\mathrm{~L}_{2}-\mathrm{M}_{4}\right.$, $4162-4260 \mathrm{eV}), 3 d_{5 / 2} \rightarrow 2 p_{3 / 2}\left(\mathrm{~L}_{3}-\mathrm{M}_{5}, 3890-3970 \mathrm{eV}\right)$, and $4 d_{3 / 2,5 / 2} \rightarrow 2 p_{3 / 2}\left(\mathrm{~L}_{3}-\mathrm{N}_{4,5}, 4435-4572 \mathrm{eV}\right)$ transitions with a total instrumental resolution of $\sim 0.65 \mathrm{eV}$ determined from the FWHM of the measured elastic peak. The transitions considered exhibit a similar qualitative behavior with the incoming photon energy and the conclusions of an analysis presented below equally apply to all our experimental data. In the following we therefore, concentrate on RIXS spectra recorded in the vicinity of the $\mathrm{I}_{3}$ edge.

For comparison, RIXS measurements of the gas-phase chloromethane $\left(\mathrm{CH}_{3} \mathrm{Cl}\right)$ were also performed at the same setup at the ID26 beamline at the ESRF. A series of $\mathrm{Cl} \mathrm{K-L}$ RIXS spectra were recorded across the energy region corresponding to resonant excitation of $\mathrm{Cl} 1 s$ electron to the lowest unoccupied molecular orbital (LUMO) $8 a_{1}$ with a total instrumental resolution of $\sim 0.55 \mathrm{eV}$.

\section{RESULTS AND DISCUSSION}

For an overview of our experimental results, it is convenient to present the recorded RIXS spectra in a contour plot as a function of the incident photon energy $\omega$ and the emitted photon energy $\omega^{\prime}$ in the absorption edge region (Fig. 1). The spectra are normalized to the photon flux, which was regularly monitored during the acquisitions. Several characteristic contributions can be distinguished in the contour plot. A well-resolved emission line dominating the RIXS spectra at the incident photon energies below $4560 \mathrm{eV}$, corresponds to the $4 d_{3 / 2,5 / 2} \rightarrow 2 p_{3 / 2}\left(\mathrm{~L}_{3}-\mathrm{N}_{4,5}\right)$ transition following the excitation I $2 p_{3 / 2} \rightarrow 15 a_{1}^{2}$ to LUMO. The cross around the incident photon energy $4559.2 \mathrm{eV}$ marks the resonant excitation energy that corresponds to the absorption maximum and to the highest intensity of the $\mathrm{L}_{3}-\mathrm{N}_{4,5}$ emission line. As the incident photon energy increases beyond the absorption maximum, the intensity of the resonant $\mathrm{L}_{3}-\mathrm{N}_{4,5}$ emission line drops and additional lower energy emission lines appear in the RIXS spectra. These tightly spaced, multipeak structures are due to resonant population of Rydberg states with a characteristic linear dispersion of the corresponding emission lines. ${ }^{11}$ Further increase of the incident photon energy leads to the dominance of the energy independent contribution, which indicates the excitation to the continuum with an onset of the fluorescent emission. The arrow around $4565 \mathrm{eV}$ shows the onset of

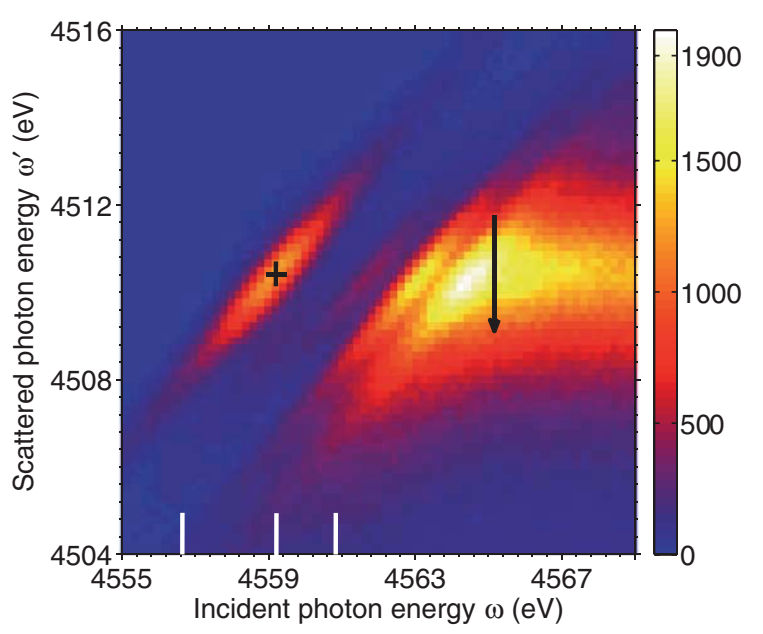

FIG. 1. Experimental RIXS spectra in $\mathrm{CH}_{3} \mathrm{I}$ recorded close to $\mathrm{I}_{3}$-edge. The cross around the incident photon energy of $4559 \mathrm{eV}$ shows the maximum of the $\mathrm{L}_{3}-\mathrm{N}_{4,5}$ emission line resulting from a resonant excitation I $2 p_{3 / 2} \rightarrow 15 a_{1}^{2}$. The arrow around $4565 \mathrm{eV}$ shows the onset of fluorescence corresponding to an approximate position of $\mathrm{CH}_{3} \mathrm{I}$ ionization threshold. Vertical lines indicate the incident photon energies used for comparison in Fig. 2.

fluorescence corresponding to an approximate position of the $\mathrm{CH}_{3} \mathrm{I}$ ionization threshold.

In order to gain an insight into the evolution of the RIXS spectra across the absorption maximum, the shape and the dispersion of the resonant $\mathrm{L}_{3}-\mathrm{N}_{4,5}$ emission line with the incident photon energy are explored. Figure 2 shows three slices through the contour plot, which present emission spectra recorded at the incident photon energies above, below, and at the resonance, as marked in Fig. 1. Let us denote the resonant absorption frequency by $\omega_{c o}$ and the transition frequency

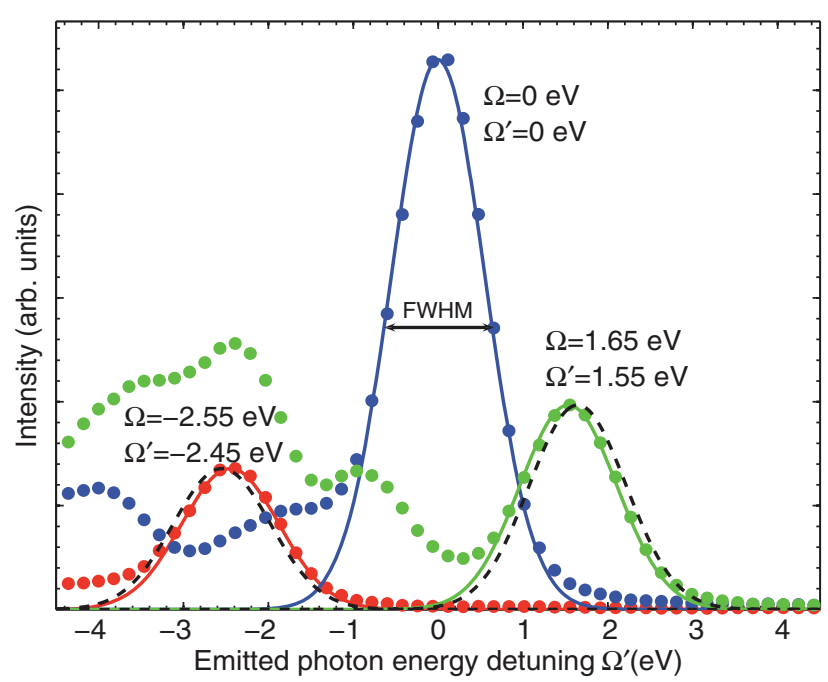

FIG. 2. Experimental RIXS spectra in $\mathrm{CH}_{3} \mathrm{I}$ recorded at the incident photon energies corresponding to resonant excitation I $2 p_{3 / 2} \rightarrow 15 a_{1}^{2}(\Omega=0 \mathrm{eV})$, above $(\Omega=1.65 \mathrm{eV})$, and below $(\Omega=-2.55 \mathrm{eV})$ the resonance energy. The spectra are plotted as a function of $\Omega^{\prime}$, the emitted photon energy relative to the peak of the $\mathrm{L}_{3}-\mathrm{N}_{4,5}$ emission line in the case of resonant excitation. The incident photon energy detuning $\Omega$ and the relative peak position of the $\mathrm{L}_{3}-\mathrm{N}_{4,5}$ emission line $\Omega^{\prime}$ are labeled in the graph. Solid lines are simulations using Eq. (2) with the parameters provided in Table I for $\mathrm{CH}_{3} \mathrm{I}$. Dashed lines show the expected positions of the emission lines in the case of linear dispersion (see text for details). 


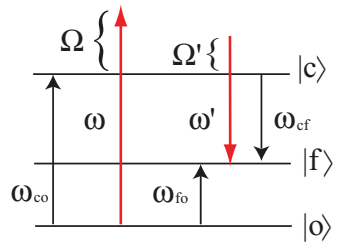

FIG. 3. A schematic diagram showing the relations between the incident and the emitted photon energies $\omega$ and $\omega^{\prime}$, the resonant absorption frequency $\omega_{c o}$, the transition frequency from the ground state to the final molecular state $\omega_{f o}$, the resonance emission energy $\omega_{c f}=\omega_{c o}-\omega_{f o}$, and the respective detunings of the incident photon energy from the resonant absorption energy $\Omega=\omega-\omega_{c o}$ and of the emitted photon energy from the resonance emission energy $\Omega^{\prime}=\omega^{\prime}-\omega_{c f}$.

from the ground state to the final molecular state by $\omega_{f o}$. The emission spectra are plotted in Fig. 2 on the relative emitted photon energy scale as a function of $\Omega^{\prime}$, where $\Omega^{\prime}=\omega^{\prime}-\omega_{c f}$ is the detuning of the emitted photon energy from the resonance emission energy $\omega_{c f}=\omega_{c o}-\omega_{f o}$ (Fig. 3). In this representation, the emission line peaking at $\Omega^{\prime}=0$ corresponds to the spectrum recorded at the resonant incident photon energy, as labeled by $\Omega=0$, where $\Omega=\omega-\omega_{\text {co }}$ is the detuning of the incident photon energy from the resonance energy $\omega_{c o}$. The emission lines recorded above and below the resonance are labeled with the positive and negative values of $\Omega$, respectively. The peak positions of the emission lines are also labeled in the graph. The uncertainty of detuning of the incident and the emitted photon energy is of the order of $10 \mathrm{meV}$, as estimated from the stability of the beamline and the spectrometer setting. From the values provided in the labels one can see that the displacement of the $\mathrm{L}_{3}-\mathrm{N}_{4,5}$ emission line with respect to the resonant line does not exactly follow the detuning of the incident photon energy from the resonant excitation, resulting in a nonlinear dispersion. The dashed lines in Fig. 2 show the expected positions of the emission lines for the linear dispersion. The deviation from the linear dispersion was observed in our earlier experiments in chlorine compounds, ${ }^{11,12}$ and the reason for the relatively weak but clearly discernible manifestation of this effect in the $\mathrm{CH}_{3} \mathrm{I}$ molecule is discussed below.

The spectral shape, in particular the width of a RIXS emission line is an essential observable, as along with the line dispersion, it carries information on the evolution of the molecular environment upon the excitation. Generally, the spectral shape of RIXS emission line depends on several factors: the lifetime broadening, the dipole transition matrix elements between molecular states, and the instrumental function. The double differential cross section for RIXS is given by the Kramers-Heisenberg formula ${ }^{15,17-19}$

$\sigma\left(\omega, \omega^{\prime}\right) \propto \sum_{c}\left|\frac{\langle f|D| c\rangle\langle c|D| o\rangle}{\omega-\omega_{c o}+i \Gamma / 2}\right|^{2} \delta\left(\omega-\omega^{\prime}-\omega_{f o}\right)$,

where $\Gamma$ denotes the FWHM of the natural lifetime broadening of the core-excited state and $D$ is the electric dipole operator. When the dipole transition matrix elements are independent of the nuclear coordinates, one may factor out the overlap of the wave functions pertaining to the nuclear degrees of freedom for each pair of states connected by the transition. Therefore, in the numerator of Eq. (1), two Franck-Condon (FC) factors appear: the first one is given by the overlap of the vibrational ground-state wave function $|o\rangle$ and the intermediate core-excited state $|c\rangle$, and the second one comes from the overlap of $|c\rangle$ and the final state $|f\rangle$.

The FC factor depends on the shape of the potential surface for both states involved in the transition. Molecular coreexcited states are often dissociative. The decay into a final dissociative state may lead to a situation, where the core-excited and the final states have nearly parallel potential surfaces. In this case the FC factor between the intermediate and the finalstate dissociative wave functions reduces to the Dirac delta function, and the expression for the RIXS cross section is given by the product of a Gaussian function describing a projection of the ground-state vibrational wave function onto the accessible continuum of vibrational wave functions in the intermediate state and a Lorentzian term, describing the electronic part of the resonant decay of the core-excited state ${ }^{19}$

$$
\sigma\left(\omega, \omega^{\prime}\right) \propto \frac{\exp \left(-\left[\left(\Omega^{\prime}-\Omega\right) / \Delta\right]^{2} \ln 2\right)}{\Omega^{\prime 2}+\Gamma^{2} / 4} .
$$

Here $\Delta$ is the half width at half maximum of the FC distribution connecting the ground and the core-excited state. For an adequate comparison with the experimental results the theoretical cross section (2) is convoluted with Gaussians describing the beamline and the x-ray spectrometer instrumental function.

The analytical expression (2) is fully applicable to the case of the $\mathrm{Cl} \mathrm{K}-\mathrm{L}$ emission in $\mathrm{HCl}$, where according to $a b$ initio calculations the dissociative potential surfaces of the core-excited and final states are nearly parallel. ${ }^{12}$ In the present case of I L-M and $\mathrm{L}-\mathrm{N}$ emission, the corresponding calculations are not available. However, using Eq. (2) as a parametric functional form we obtain a good quantitative fit of the experimental $\mathrm{L}_{3}-\mathrm{N}_{4,5}$ emission line as demonstrated in Fig. 2. Therefore, in the following we assume that the intermediate and the final-state dissociative potentials are parallel.

In order to explore the evolution of the $\mathrm{L}_{3}-\mathrm{N}_{4,5}$ emission line across the resonance we monitor the position of the peak intensity and the FWHM of the line with the incident photon energy. Figure 4 shows the dispersion and the FWHM of the $\mathrm{L}_{3}-\mathrm{N}_{4,5}$ emission line in $\mathrm{CH}_{3} \mathrm{I}$ and of the $\mathrm{Cl} \mathrm{K}-\mathrm{L}$ emission line in $\mathrm{CH}_{3} \mathrm{Cl}$ as a function of the incident photon energy detuning from the resonance. The error bars indicated on the graphs in Fig. 4 are related to the extraction of the dispersion and the FWHM values from the experimental spectra. We obtain an excellent quantitative agreement of the experimental data with the results of the simulations using Eq. (2). However, a qualitatively different behavior is observed for $\mathrm{CH}_{3} \mathrm{I}$ as compared to $\mathrm{CH}_{3} \mathrm{Cl}$. While in $\mathrm{CH}_{3} \mathrm{Cl}$ dispersion of the peak position is strongly nonlinear around the resonance, $\mathrm{CH}_{3} \mathrm{I}$ shows a small deviation from the linear dispersion [Fig. 4(a)]. This is further illustrated in Fig. 4(b) where the peak position is plotted with respect to the linear dispersion, and a deviation from the zero indicates the presence of nonlinear dispersion in both molecules. The FWHM of the emission line exhibits a significant narrowing at the resonance in $\mathrm{CH}_{3} \mathrm{Cl}$, whereas in $\mathrm{CH}_{3} \mathrm{I}$ the line width hardly changes across the 

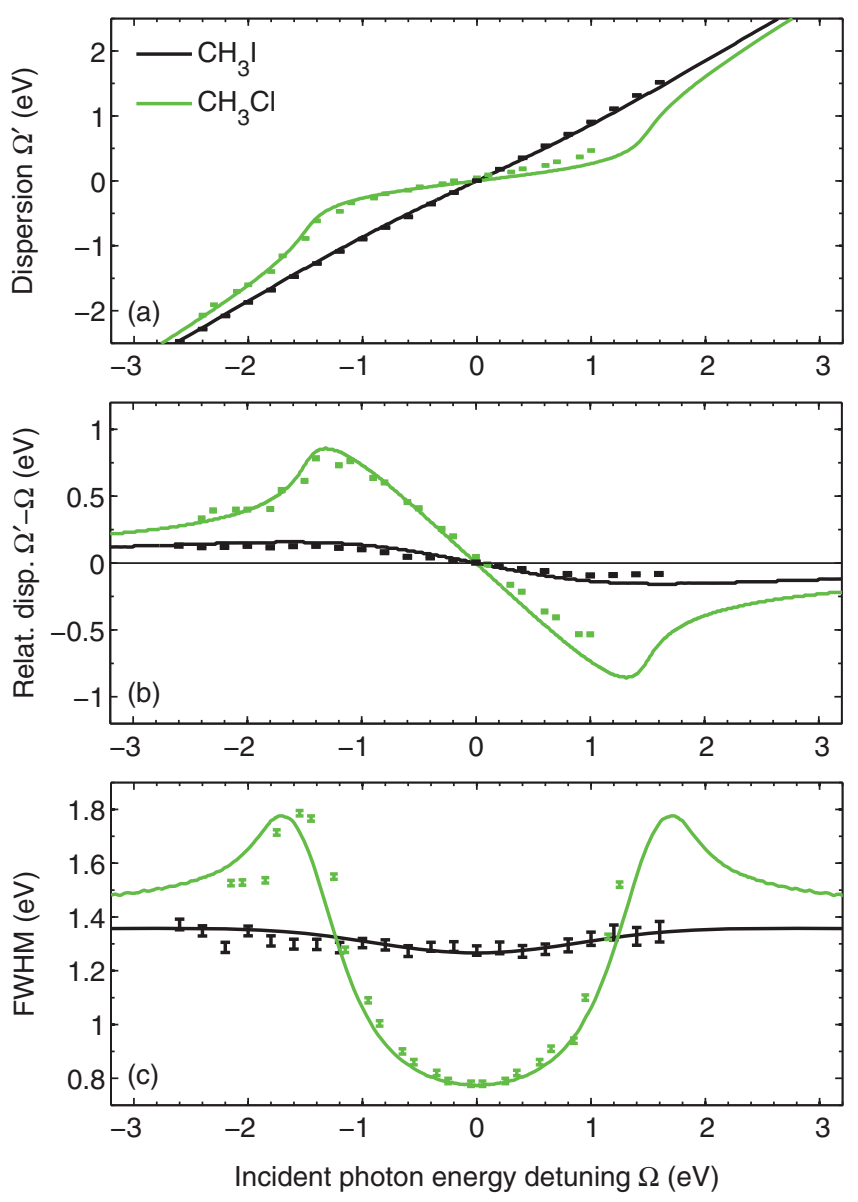

FIG. 4. (a) Dispersion, (b) dispersion relative to the linear dispersion, and (c) FWHM of the $\mathrm{I}_{3}-\mathrm{N}_{4,5}$ (black) and $\mathrm{Cl} \mathrm{K}-\mathrm{L}$ in $\mathrm{CH}_{3} \mathrm{Cl}$ (green) emission lines as a function of the incident photon energy detuning with respect to the resonance energy. Solid lines show simulations using Eq. (2) for RIXS cross section convoluted with the beamline and the spectrometer instrumental function. The error bars are related to the extraction of the dispersion and the FWHM values from the experimental spectra.

resonance [Fig. 4(c)]. In other words, RIXS spectral features are much less affected by the resonant conditions in $\mathrm{CH}_{3} \mathrm{I}$ as compared to $\mathrm{CH}_{3} \mathrm{Cl}$.

The nature of the qualitatively different behavior in the case of $\mathrm{CH}_{3} \mathrm{I}$ can be revealed upon a close examination of the parameters used in the simulations of the RIXS emission lines. The values of the lifetime broadening, ${ }^{20}$ the resonant excitation energy, and the instrumental resolutions are summarized in Table I. Note that the values of the FC factor were determined by fitting Eq. (2) to the experimental data. The RIXS spectral features show a strong sensitivity to the FC factor providing a fitted value with an estimated error of less than $5 \%$. Thus, within the limits of applicability of Eq. (2) the value of the FC factor can be determined with a reasonable accuracy from the experimental RIXS data without a necessity to perform challenging ab initio calculations.

In the $\mathrm{CH}_{3} \mathrm{Cl}$ molecule the width of the vibrational broadening is larger than the width of the natural lifetime broadening of the $\mathrm{Cl} \mathrm{K}$-shell core-hole. In this regime the shape of RIXS emission line away from the resonance is dominated by the dynamical broadening reflected by the FC distribution. Near the resonance, however, the dynamical broadening is
TABLE I. The parameters used in the simulations presented in Figs. 2 and 4. The lifetime broadening values are taken from Ref. 20, the values of the FC factor are determined from the fit.

\begin{tabular}{lcc}
\hline \hline & $\mathrm{CH}_{3} \mathrm{Cl}$ & $\mathrm{CH}_{3} \mathrm{I}$ \\
\hline Resonance energy, $\omega_{c o}$ & $2822.8 \mathrm{eV}$ & $4559.2 \mathrm{eV}$ \\
Lifetime broadening, $\Gamma$ & $0.7 \mathrm{eV}$ & $2.8 \mathrm{eV}$ \\
Lifetime, $\tau=\hbar / \Gamma$ & $0.94 \mathrm{fs}$ & $0.23 \mathrm{fs}$ \\
FC FWHM, $2 \Delta$ & $1.3 \mathrm{eV}$ & $1.2 \mathrm{eV}$ \\
$\Gamma / 2 \Delta$ & 0.54 & 2.3 \\
Beamline resolution FWHM & $0.4 \mathrm{eV}$ & $0.14 \mathrm{eV}$ \\
Spectrometer resolution FWHM & $0.4 \mathrm{eV}$ & $0.64 \mathrm{eV}$ \\
\hline \hline
\end{tabular}

quenched and the width of the line is determined by the natural lifetime broadening, resulting in a significant line narrowing at the resonance energy. In the case of the $\mathrm{CH}_{3} \mathrm{I}$ molecule, the natural lifetime broadening of the I L-shell core-hole is about two times larger than the FC width. Under such conditions the quenching of vibrational broadening at the resonance has no strong effect on the line narrowing.

The time-independent and the time-dependent descriptions of resonant Raman scattering (radiative or nonradiative) are equivalent and provide equally good agreement with the experimental observations. ${ }^{15}$ However, the physical meaning of the nonlinear dispersion of the emission lines becomes transparent if we consider the dynamical aspect of the RIXS process. Generally, the relaxation of a core-hole upon resonant excitation competes with the process of nuclear rearrangement triggered by the photon absorption. This interplay can be described using a concept of an effective scattering time $\tau_{\text {eff }}=1 / \sqrt{\Omega^{2}+\Gamma^{2}}$ (Refs. 14 and 15). The effective time delay of the photon emission with respect to photon absorption takes the maximum value of $\Gamma^{-1}$ at the resonant energy, where $\Omega=0$. Therefore, the largest effect of the nuclear dynamics is expected at photon energy tuned to the resonance energy, where the scattering time is long enough to allow for the nuclear rearrangement to take place before the core-hole decay. In this case, a part of the excitation energy is transferred into the nuclear motion, which is manifested by the nonlinear dispersion. Away from the resonance the scattering time is shortened, the nuclei are effectively frozen, and the linear dispersion of the emission line is recovered. A deviation from linear dispersion is strongly pronounced in the $\mathrm{CH}_{3} \mathrm{Cl}$ data (Fig. 4), where the $\sim 1$ fs natural lifetime of the $\mathrm{Cl}$ $\mathrm{K}$-shell core hole allows for the occurrence of considerable nuclear dynamics. However, in the case of the $\mathrm{CH}_{3} \mathrm{I}$ molecule, no significant nuclear motion can occur in the time interval determined by the iodine L-shell core-hole lifetime ( $0.23 \mathrm{fs})$. The scattering time is short even for $\Omega=0$, and practically no energy can be transferred to the nuclei before the photon is emitted. As a consequence, only a slight deviation of the emission line from the linear dispersion is expected.

The variations of line shapes, widths, and positions observed in our measurements are qualitatively similar to the ones resulting from convolutions of Gaussian spectral and spectrometer functions with a core-excited resonance having a Lorentzian profile, common to atomic, molecular, and solid-state resonant Raman spectra. ${ }^{21}$ However, in the present 

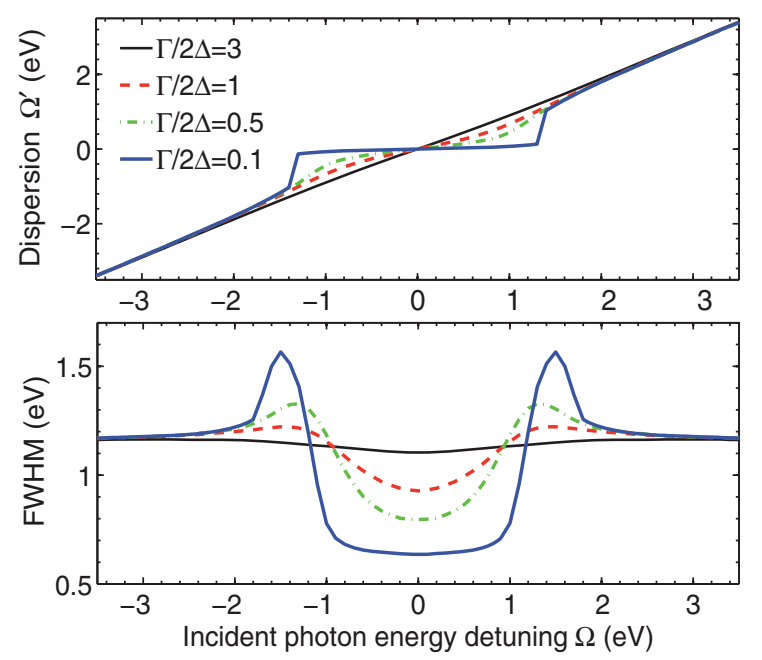

FIG. 5. Calculated relative peak position and FWHM of RIXS emission lines as a function of the incident photon energy detuning with respect to the resonant excitation. The curves are calculated at various ratios of lifetime broadening to the FC factor using Eq. (2) for RIXS cross section with FC FWHM $2 \Delta=1 \mathrm{eV}$, convoluted with the instrumental function with $\mathrm{FWHM}=0.6 \mathrm{eV}$.

measurements the instrumental contribution to the spectra is rather small (on the order of $10 \%$ and $2 \%$ for $\mathrm{CH}_{3} \mathrm{Cl}$ and $\mathrm{CH}_{3} \mathrm{I}$, respectively) due to the narrow spectral bandwidth of the incident radiation and relatively large natural widths. Therefore, the observed nonlinear dispersion and the line width modulation are largely due to the dynamical effects, accounted for by the Gaussian term in Eq. (2) for the RIXS cross section and are specific to the dissociative processes in molecules.

A good quantitative agreement obtained between the experiment and the fit based on the approximate parameterization encourages the use of Eq. (2) to illustrate the role of the natural lifetime in description of the RIXS spectra. Figure 5 shows the emission line dispersion and the corresponding FWHM as a function of the incident photon energy detuning for a series of emission lines calculated using Eq. (2) for various ratios of lifetime broadening $\Gamma$ to $F C$ factor. The cross sections are convoluted with the Gaussian instrumental function with FWHM equal to $0.6 \mathrm{eV}$. The value of the $\mathrm{FC}$ factor is kept constant $(2 \Delta=1 \mathrm{eV}$ FWHM). One can see that for a relatively long-lived inner hole, where $\Gamma / 2 \Delta<1$, the dispersion is strongly nonlinear and the linewidth of the RIXS emission line experiences a substantial narrowing around the resonance. This regime, characteristic of the $\mathrm{Cl} \mathrm{K}$-shell excitation, corresponds to the occurrence of significant nuclear dynamics in a molecule. When $\Gamma / 2 \Delta>1$, the dispersion tends to a quasilinear dependence and a modulation of a RIXS line width across the resonance is strongly reduced. In this regime, characteristic of the I L-shell excitation, an ultrashort natural lifetime limits the onset of nuclear dynamics and the FWHM of a RIXS line is only weakly affected by quenching of the vibrational broadening. Note that although the variation of the FWHM value around the resonance falls below $10 \%$ at $\Gamma / 2 \Delta$ $=3$, the observable narrowing can serve as an indicator of a dynamic process taking place in a molecule on the time scale determined by the natural lifetime of $\sim 0.2 \mathrm{fs}$. Thus, an ultrafast molecular dynamics is revealed through a subtle modula- tion of RIXS spectral features, particularly by the narrowing of the emission line in the resonance energy region.

\section{CONCLUSIONS}

Resonant Raman scattering can be equally well described using both the time-independent and the time-dependent approaches. In this paper we aim to demonstrate how the highresolution spectroscopic measurements can provide insight into the ultrafast molecular dynamics, using a link between the energy- and time-domain measurements that provides a clear physical interpretation of the problem. Here we discuss sensitivity of RIXS spectroscopy to small variations in molecular geometry upon photon absorption on a subfemtosecond time scale determined by the core-hole lifetime.

To conclude, we have measured a series of RIXS spectra of the $\mathrm{CH}_{3} \mathrm{I}$ molecule in the hard-X-ray region near the iodine $\mathrm{L}_{2}$ and $\mathrm{L}_{3}$ absorption edges. A previously unexplored regime characterized by an ultrashort natural lifetime of an iodine L-shell core-hole reveals dispersive RIXS spectral features that were recently recognized as a fingerprint of dissociative molecular states. It was shown that the nuclear dynamics induced in a molecule upon core-hole excitation and reflected in the RIXS spectra, is limited by the time scale imposed by the natural lifetime of the core hole. In the case of the $\mathrm{CH}_{3} \mathrm{I}$ molecule, the natural lifetime of $\sim 200$ attoseconds of the iodine L-shell core-hole sets a limitation to the occurrence of nuclear dynamics. Nevertheless, a slight deviation of the RIXS spectral features around the resonance observed in $\mathrm{CH}_{3} \mathrm{I}$ demonstrates a large sensitivity of RIXS spectroscopy to nuclear rearrangement in a molecule, extending its application to the subfemtosecond time range.

\section{ACKNOWLEDGMENTS}

We acknowledge the ID26 beamline staff for assistance in the experiment. This work is supported by the Slovenian Research Program P1-0112. The financial support of the Swiss National Science Foundation is also acknowledged.

${ }^{1}$ A. Kotani and S. Shin, Rev. Mod. Phys. 73, 203 (2001).

${ }^{2}$ P. Skytt, P. Glans, J.-H. Guo, K. Gunnelin, C. Såthe, J. Nordgren, F. Kh. Gel'mukhanov, A. Cesar, and H. Ågren, Phys. Rev. Lett. 77, 5035 (1996).

${ }^{3}$ P. Glans, K. Gunnelin, P. Skytt, J. H. Guo, N. Wassdahl, J. Nordgren, H. Agren, F. Gel'mukhanov, T. Warick, and E. Rotenberg, Phys. Rev. Lett. 76, 2448 (1996).

${ }^{4}$ D. W. Lindle, P. L. Cowan, R. E. LaVilla, T. Jach, R. D. Deslattes, B. Karlin, J. A. Sheehy, T. J. Gil, and P. W. Langhoff, Phys. Rev. Lett. 60, 1010 (1988).

${ }^{5}$ S. H. Southworth, D. W. Lindle, R. Mayer, and P. L. Cowan, Phys. Rev. Lett. 67, 1098 (1991).

${ }^{6}$ J. D. Mills, J. A. Sheehy, T. A. Ferrett, S. H. Southworth, R. Mayer, D. W. Lindle, and P. W. Langhoff, Phys. Rev. Lett. 79, 383 (1997).

${ }^{7}$ R. Guillemin, S. Carniato, W. C. Stolte, L. Journel, R. Taieb, D. W. Lindle, and M. Simon, Phys. Rev. Lett. 101, 133003 (2008).

${ }^{8}$ S. Carniato, R. Guillemin, W. C. Stolte, L. Journel, R Taïeb, D. W. Lindle, and M. Simon, Phys. Rev. A 80, 032513 (2009).

${ }^{9}$ J. Hoszowska, A. K. Kheifets, J.-Cl. Dousse, M. Berset, I. Bray, W. Cao, K. Fennane, Y. Kayser, M. Kavčič, J. Szlachetko, and M. Szlachetko, Phys. Rev. Lett. 102, 073006 (2009).

${ }^{10}$ M. Kavčič, M. Žitnik, K. Bučar, A. Mihelič, M. Štuhec, J. Szlachetko, W. Cao, R. Alonso Mori, and P. Glatzel, Phys. Rev. Lett. 102, 143001 (2009). 
${ }^{11}$ M. Kavčič, M. Žitnik, K. Bučar, A. Mihelič, S. Carniato, L. Journel, R. Guillemin, and M. Simon, Phys. Rev. Lett. 105, 113004 (2010).

${ }^{12}$ M. Simon, L. Journel, R. Guillemin, W. C. Stolte, I. Minkov, F. Gel'mukhanov, P. Sałek, H. Ågren, S. Carniato, R. Taïeb, A. C. Hudson, and D. W. Lindle, Phys. Rev. A 73, 020706 (2006).

${ }^{13}$ L. Journel, L. El Khoury, T. Marin, R. Guillemin, S. Carniato, A. Avila, R. Delaunay, C. F. Hague, and M. Simon, Rev. Sci. Instrum. 80, 93105 (2009).

${ }^{14}$ F. Gel'mukhanov, P. Sałek, T. Privalov, and H. Ågren, Phys. Rev. A 59, 380 (1999)
${ }^{15}$ F. Gel'mukhanov and H. Ågren, Phys. Rep. 312, 87 (1999).

${ }^{16}$ M. Žitnik, M. Kavčič, K. Bučar, and A. Mihelič, Nucl. Instrum. Methods Phys. Res. B 267, 221 (2009).

${ }^{17}$ F. Gel'mukhanov, T. Privalov, and H. Ågren, Phys. Rev. A 56, 256 (1997).

${ }^{18}$ P. Sałek, F. Gel'mukhanov, and H. Ågren, Phys. Rev. A 59, 1147 (1999).

${ }^{19}$ F. Gel'mukhanov and H. Ågren, Phys. Rev. A 54, 379 (1996).

${ }^{20}$ J. L. Campbell and T. Papp, At. Data Nucl. Data Tables 77, 1 (2001).

${ }^{21}$ G. B. Armen and H. Wang, Phys. Rev. A 51, 1241 (1995). 